\title{
COMENTARIOS EN TORNO A BUNGE, MARGAIN Y LA PARADOJA
}

JOSÉ A. ROBLES

Universidad Nacional

Autónoma de México

La historia se desarrolla en tres números previos de Critica; en ellos, el asunto se presenta (Bunge: III, 9, pp. 27-29), se discute (Margäin: VI, 18, pp. 113-116) y se responde (Bunge: VII, 20, pp. 105-107). El motivo de la discordia: "The Paradox of Addition and its Dissolution", nota del Dr. Bunge que el lector interesado puede encontrar en el número 9 de Crítica, antes citado, pp. 27-29. Dado que la historia no es ni muy larga ni muy remota, me concretaré a considerar sólo los últimos acontecimientos de la misma.

Bunge, en su respuesta a Margáin (Crítica, VII, 20, p. 105), nos dice:

Pero lo importante, y ésta es la tesis de mi nota, es que el principio de adición tolera inferencias ilegítimas, tales como

México está en América.

$\therefore$ México está en América o no tengo ganas de pensar.

$\therefore$ Si tengo ganas de pensar, México está en América.

Esta es una falacia porque la conclusión afirma el error consistente en que, para que yo tenga ganas de pensar, es necesario que México esté en América (o, lo que es equivalente, basta que yo tenga ganas de pensar para que México se ubique en América).

En este punto uno mi queja a la de Margáin (Crítica, VI, 18) para señalar que las cosas no son tan claras ni tan directas como las señala Bunge. Nos detendremos un momento a considerar su argumento valiéndonos de las siguientes convenciones: 


$$
\begin{aligned}
& p=\text { "México está en América" } \\
& q=\text { "Tengo ganas de pensar" }
\end{aligned}
$$

entonces, podemos presentar el argumento de Bunge median. te el siguiente conjunto de enunciados: ${ }^{1}$

$$
\text { (A) } \begin{aligned}
& p \vdash(p \vee-q) \\
& p \vdash(q \supset p)
\end{aligned}
$$

pero, igualmente y de manera legítima podríamos haber tenido el siguiente conjunto:

$$
\text { (B) } \begin{aligned}
& p \vdash(p \vee q) \\
p & \vdash(-q \supset p)
\end{aligned}
$$

$\mathrm{Y}$ así, tanto $q$ como $-q$ son condiciones suficientes de la verdad de p. Esto es, no importa qué proposición, como antecedente, le antepongamos a $p$ para obtener un condicional verdadero, pero con la condición de que p sea una premisa del sistema de proposiciones que estemos considerando. Así, concluimos que lo que nos dice Bunge ("Esta es una falacia ...") sólo puede mantenerse si nos olvidamos de la premisa de la que partimos, i.e, p. Aquí podemos añadir que ciertamente es un error pensar que $q$ es una condición suficiente de la verdad de $p$ porque, en último análisis, y dentro de este ejemplo (generalizándolo de la manera usual), $q$ (o bien $-q$ ) es condición suficiente de:

1 La formulación que da Bunge del principio de adición $(P A)$ en su respuesta a Margáin difiere de la formulación en su artículo primero en que, en el primer caso, el $P A$ se formula en términos de una regla de derivación:

$$
\alpha \vdash(\alpha \vee \beta)
$$

en tanto que en el último, la formulación era:

$$
\vdash(\alpha \supset(\alpha \vee \beta))
$$

así que, puesto que hay una diferencia mínima, en cuanto a los puntos que se presentan, debida a la formulación, en la primera parte de mi escrito, mutatis mutandi, me temo repetir gran parte de la argumentación de Margáin, por lo que no me detendré mucho en ello. Cf. Crítica, VI, 18, pp. 113-116. 


$$
p v-p
$$

ya que partiendo de la segunda expresión de (A) (o bien de la segunda de (B) con las modificaciones obvias) y aplicando el Teorema de Deducción, obtenemos:

$$
\vdash(p \supset(q \supset p))
$$

$\mathrm{y}$, finalmente, vía transformaciones elementales:

$$
\vdash(q \supset(p \vee-p)) \text {. }
$$

Pero, entonces, obviamente cualquier otra proposición que tengamos en lugar de $q$ será condición suficiente de la verdad de un teorema de lógica. En el ejemplo de Bunge el error consiste en considerar que su conclusión se deriva del conjunto vacío de premisas, esto es, en olvidar que $p$ funge como la premisa (única en este caso) del argumento que nos presenta.

Algo similar sucede en cualquier sistema de lógica mo$\mathrm{dal}^{2}$ en el que tengamos, como regla para introducir el operador de necesidad ' $N$ ', la siguiente:

$$
\vdash p \Rightarrow \vdash N p \text {. }
$$

Usando esta regla, y por pasos elementales, obtenemos:

$$
\begin{aligned}
& \vdash p \\
& \vdash N_{p} \\
& \vdash(q \supset N p)
\end{aligned}
$$

y esto último lo podemos interpretar diciendo que cualquier proposición $q$ implica la necesidad de $p$, porque hemos afirmado previamente que la necesidad de $p$ es una tesis del sistema. Así, tanto en un sistema extensional de lógica clásica, como en sistemas modales, podemos tener el $P A$ sin que

2 Bunge detectó originalmente la paradoja en sistemas de enunciados modales, indicando que la misma se generaba, igualmente, en sistemas de enunciados imperativos y en lógica clásica de primer orden. Cf. Crítica, III, 9. 
surjan, según creo haber mostrado, molestas paradojas del tipo que Bunge nos señala.

El Dr. Bunge podría quizá aceptar los puntos anteriores y negar que le sean aplicables ya que, a continuación de lo que previamente he citado de él (supra, p. 105), nos dice:

La falacia consiste en ampliar el universo del discurso originario (la clase de referencia de $p$ ) agregando la clase de referencia de $q$. Este paso, sintácticamente legítimo, puede no serlo semánticamente, ya que, si introduce irrelevancias, puede causar confusión o error.

En este punto me gustaría responder que el riesgo puede minimizarse una vez que se señalan los peligros que pueda ocultar cierta manera de interpretar alguna forma específica de argumento; pero insisto que, en el caso presente, la forma de argumento (el $P A$ ) por sí misma es perfectamente legítima tanto sintáctica como semánticamente y que el error puede derivarse sólo de una forma parcial de considerarla. Pero éste es un riesgo que podemos tener casi en cualquier caso. Por otra parte, no quiero insistir demasiado sobre el punto de irrelevancias, ya que creo que el mismo podrá adjudicarse sólo una vez que tengamos criterios aceptables para eliminarlas, los cuales pueden resultar excesivamente complicados de formular y, mucho me temo, serán, en la mayoría de los casos, muy ad hoc. Sin embargo, hay un segundo punto en el artículo de Bunge con el que mis problemas son mayores.

En su respuesta (Crítica, VII, 20, pp. 106-7), Bunge nos dice: ${ }^{3}$

La segunda manera de evitar las falacias semánticas cometidas con la complicidad del principio de adición es

3 Hay un primer caso que Bunge considera para evitar la introducción de irrelevancias en sistemas de enunciados. Pero él mismo no lo enfatiza mucho. por lo que no lo consideraré en esta nota; nos dice: "Hay dos maneras de evitar estas falacias. Una es trabajar en contextos axiomáticos... Pero ta] limitación, deseable en la etapa final de la fundamentación, sería paralizante en la etapa constructiva" (Crítica, VII, 20, p. 106). 
montar guardia para evitar el contrabando de predicados extraños al universo del discurso originario. Esto es lo que se hace, por ejemplo, al enunciar con algún cuidado el teorema de interpolación de Craig: "Si $p$ implica a $q$, entonces existe una proposición $r$, que no contiene predicados diferentes de los que figuran en $p y q$, y tal que $p$ implica a $r$, y $r$ implica a $q . "$ La cláusula subrayada expresa la condición que he llamado de clausura (u homogeneidad) semántica.

Antes de pasar al punto que me interesa, quisiera reformular, ligeramente, el Teorema de Craig $(T C)$ y así poner de manifiesto algunos puntos que me parecen pertinentes para la discusión. En la reformulación tomo en cuenta, básicamente, el siguiente resultado obvio:

$$
\text { (C) } \vdash(p \supset q) \Rightarrow \operatorname{Pr}(p) \cap \operatorname{Pr}(q) \neq \phi \mathrm{v} \vdash-p \mathrm{v} \vdash q \text {. }
$$

donde

$$
\operatorname{Pr}(\alpha)={ }_{\mathrm{df}} \text { conjunto de predicados en } \alpha \varepsilon \text { Fmla }
$$

y

Fmla $=_{\mathrm{d} f}$ conjunto de fórmulas de primer orden del sistema que se considera.

Lo que (C) nos dice es que: Si "si $p$ entonces $q$ " es un teorema $y p$ no es una contradicción $y q$ no es una tesis del sistema, entonces hay al menos una letra predicativa (predicado) que figura tanto en $p$ como en $q$. Lo que el $T C$ nos dice es que si la hipótesis de (C), en los términos en que acabo de presentarla, se cumple, entonces hay una $r \varepsilon$ Fmla en la que figuran sólo los predicados comunes a $p$ y $q$ y, además, que

$$
\vdash(p \supset r) \text { y } \vdash(r \supset q)
$$

son verdaderas.

Para la discusión que sigue permítaseme pues, a riesgo 
de enfadar a los lectores, presentar claramente los supues. tos $\operatorname{del} T C$ :

$$
\begin{aligned}
& (1) \quad \vdash(p \supset q) \\
& \text { y (2) } \not-p \\
& \text { y (3) } \not q q
\end{aligned}
$$

de esto, Craig concluye:

(4) $\exists r \varepsilon$ Fmla : $\operatorname{Pr}(r) \subseteq(\operatorname{Pr}(p) \cap \operatorname{Pr}(q)) \& \vdash(p \supset r) \&$ $\vdash(r \supset q)$.

Una vez con los puntos anteriores a la mano, puedo presentar mi argumento en los siguientes términos:

Lo que sostengo es que apelar al TC en el presente contexto no es pertinente por las siguientes razones:

(1) El Dr. Bunge nos presenta un caso de derivación a partir de premisas y acerca de esto el $T C$ no especifica o impone restricciones acerca de posibles fórmulas de interpolación. Ciertamente, en el ejemplo de Bunge:

$$
p \vdash(q \supset p)
$$

cualquier $r \varepsilon$ Fmla, tenga o no predicados comunes con $p$ y/o con $q$ y tal que

$$
\vdash r
$$

o bien tal que $r$ sea una premisa del sistema de fórmulas que consideramos, es tal que

$$
p,(r) \vdash(q \supset r) \text { y } p,(r) \vdash(r \supset p)
$$

(donde " $r$ " representa o bien un teorema de lógica o bien una premisa del sistema y desaparece de la lista de premisas obviamente en el primer caso mas no en el segundo), esto es, $r$ puede ser una fórmula de interpolación; por otra parte, notemos que tal $r$ es una fórmula plenamente relevante al sistema en tanto que puede ser cualquier premisa del mismo, 
pero es por completo irrelevante (conforme a otras conside. raciones) cuál premisa escojamos para interpolar.

(2) Aun en el caso en que tuviéramos

$$
\vdash(q \supset p)
$$

esto es que ${ }^{\ulcorner}(q \supset p)^{\top}$, fuera un teorema de lógica, si además,

$$
\text { (a) } \vdash p
$$

o bien

$$
\text { (b) } \vdash-q
$$

entonces, cualquier $r \varepsilon$ Fmla, tal que

$$
\left(a^{\prime}\right) \vdash r
$$

o bien

$$
\left(b^{\prime}\right) \vdash-r
$$

(respectivamente) tenga o no predicados comunes con $p \mathrm{y} / \mathrm{o}$ con $q$, sería una fórmula de interpolación, pues claramente

$$
\vdash(q \supset r) \text { y } \vdash(r \supset p)
$$

son ambos enunciados verdaderos; ahora bien, si se cumplieran conjuntamente las cláusulas (a) y (b), entonces cual. quier $r \varepsilon$ Fmla, sin más condiciones, sería una adecuada fórmula de interpolación.

(3) Por último, es posible introducir en un sistema, vía $P A$, fórmulas que por alguna razón se consideren irrelevantes al sistema y, posteriormente, tener esas mismas fórmulas como adecuadas fórmulas' de interpolación, conforme al $T C$, de la siguiente manera:

Supongamos que $r \varepsilon$ Fmla sea tal que cumpla con las condiciones (2) y (3) del TC (cf. supra, p. 110), irrelevante dentro del contexto que se considera y tal que no tenga un solo 
predicado común con alguna otra $p \varepsilon$ Fmla tal que esta última sea una premisa en nuestro sistema, entonces:

$$
p \vdash(-r \vee p) \quad(\text { por } P A)
$$

y por pasos proposicionales aceptados, obtenemos:

$$
\begin{aligned}
& \vdash(p \supset(r \supset p)) \\
\therefore & \vdash(r \supset(p \supset p)) \\
\therefore & \vdash(r \supset(-(p \supset p) \supset r)) \\
\therefore & \vdash(-(r \supset-p) \supset(-p \supset r))
\end{aligned}
$$

y para este último caso, que podemos perfectamente suponer que cumple con las tres condiciones señaladas en la hipótesis del TC (supra, p. -), tenemos como una posible fórmula de interpolación justamente a $r$, la fórmula considerada como irrelevante en un principio, pues es claro que:

$$
\vdash(-(r \supset-p) \supset r) \quad \mathrm{y} \quad \vdash(r \supset(-p \supset r))
$$

son, ambos, enunciados verdaderos.

Lo que todo lo anterior muestra, según creo, es que si se introducen 'irrelevancias' (vía $P A$ o por cualquier otro camino legítimo) en un sistema de fórmulas, aquéllas ya estarán presentes en el mismo antes de que podamos apelar al $T C$ o bien sin que podamos apelar a éste para detectarlas o eliminarlas. Dado que el mismo Bunge considera que sería dar un paso demasiado radical eliminar el $P A^{4}$ y puesto que, según he señalado, no es mediante el $T C$ como podemos de. tectar las irrelevancias dentro de un sistema de fórmulas, entonces no veo cuál sea la solución que Bunge nos ofrece para su problema.

La discusión previa espero que ponga claramente de manifiesto en dónde veo oscuridades en la solución de Bunge a

4 Él nos dice: "Es obvio que hay que remediar esta situación. Es igualmente claro que el remedio no consiste en extirpar el principio, puesto que se lo necesita diariamente..." (Ibid.). 
la supuesta paradoja y las razones de esto. La conclusión ya la he señalado: no veo que el $P A$ esté mal (ni sintáctica ni semánticamente) tal como está y de esto se sigue que, cuando menos en el caso aquí planteado, no veo que haya algún problema con el mismo; y tampoco veo cómo el TC nos auxilie para impedir que surjan enunciados irrelevantes dentro de un sistema de enunciados que puede ya contener a aquéllos. ${ }^{5}$

${ }^{5}$ En conversación, el Dr. Bunge me indicó amablemente puntos básicos de 'desacuerdo con mi nota. Esta la publico sin modificaciones, sin embargo, por dos razones: (a) aun no veo la fuerza de la respuesta de Bunge a Margáin; (b) otras personas, cuya opinión acerca de los puntos que toca Bunge es también respetable, han coincidido en aceptar el punto de vista expresado en esta nota. Esto me hace concluir que el punto que Bunge formula acerca del principio de adición, si no es incorrecto, sí se presenta de manera que puede confundir a sus lectores. Aclarar esto redundaría en un beneficio común. 